\title{
DETERMINAÇÃO DOS PARÂMETROS REOLÓGICOS DA SUSPENSÃO DA MICROALGA CHLORELLA SP
}

\author{
P.H.G. VINHAL* ${ }^{*}$ M.S. CRISTIANINI, H.S. SAADE, D.H.P. GUIMARÃES, C.C. LOURES, \\ M.B. SILVA
}

Escola de Engenharia de Lorena, Universidade de São Paulo, Departamento de Engenharia Química

*e-mail: Pedro.hvinhal@gmail.com

\begin{abstract}
RESUMO
A produção de combustíveis renováveis obteve um grande impulso nos últimos anos, com o aumento do preço dos combustíveis e as crescentes preocupações com questões ambientais. Dentre as diversas matérias primas disponíveis na produção de biocombustíveis, as microalgas surgem como uma alternativa sustentável devido à elevada produtividade e à falta de necessidade de solos produtivos e água com qualidade. Levando em conta o interesse crescente na utilização das microalgas como matéria prima na produção do biodiesel, o objetivo do presente trabalho foi determinar os parâmetros reológicos de suspensões da microalga Chlorella $s p$ em diferentes tempos de cultivo, sob seis diferentes condições de temperatura, salinidade e níveis de $\mathrm{CO}_{2}$, $\mathrm{NO}_{3}$ e $\mathrm{PO}_{4}$, de modo a, posteriormente, estimar as demandas energéticas de cada etapa. Para cada uma dessas condições foram feitos testes em reômetro rotacional de cilindros concêntricos (Brookfield, LVDV). Os resultados mostraram que a suspensão da microalga Chlorella $s p$ trata-se de um material com características dilatantes, visto que o incide de comportamento, em todos os casos, resultou em valores superiores a 1.
\end{abstract}

\section{INTRODUÇÃO}

A crise do petróleo, nas décadas de 70 e 80 , suscitou diversos estudos referentes à pirólise de triglicerídeos. Com o risco de esgotamento de algumas fontes de energia, especialmente a energia fóssil que não apresenta perspectivas de renovação, há uma motivação para o desenvolvimento de tecnologias que permitam utilizar fontes renováveis de energia. Ademais, a crescente preocupação da sociedade com questões ambientais deve influir nas decisões dos dirigentes quanto às possibilidades de utilização das fontes energéticas. Dentro deste aspecto, os combustíveis fósseis, tais como o óleo díesel e a gasolina são os mais criticados, devido à produção de uma quantidade de $\mathrm{CO}_{2}$ que o planeta não tem condições de assimilar a longo prazo, causando o chamado efeito estufa, e também pela possibilidade de emissão de óxidos de enxofre. Tais combustíveis pertencem à categoria dos combustíveis não biodegradáveis que, durante o uso dos mesmos, emitem compostos poluentes à atmosfera (Azeredo, 2012).

$O$ biodiesel, por se tratar de um combustível derivado de óleos vegetais e gorduras animais, é um combustível biodegradável, com baixa emissão de compostos poluentes na atmosfera, podendo, desta maneira, ser considerado o combustível 
do futuro. Estimulados por um catalisador, tais óleos reagem quimicamente com álcool. Existem diferentes espécies de oleaginosas no Brasil que podem ser usadas para produzir o biodiesel. Entre elas estão a mamona, dendê, canola, girassol, amendoim, soja e algodão. Matérias-primas de origem animal, como o sebo bovino e gordura suína, também podem ser utilizadas na fabricação do biodiesel (Costa Neto, 2000; Ferrari et al., 2005; Albuquerque, 2006; Dantas, 2006; Barbosa, 2007; Sharma, 2008; Silva Filho, 2010; Basha, 2009; Fitzpatrick et al., 2010; Dogaris et al., 2013).

No entanto, projetos experimentais realizados em todo o mundo têm destacado as microalgas como fonte de produção do biodiesel. Estes organismos apresentam inúmeras vantagens sobre as culturas terrestres tradicionais na produção de biocombustíveis, tais como a elevada eficiência na conversão da luz solar em biomassa, a possibilidade de cultivo em terrenos impróprios para culturas alimentares e de utilização de águas não potáveis para a produção de biomassa, a acumulação de lipídios, capacidade de sorção de metais pesados ( $\mathrm{Hg}, \mathrm{Zn}, \mathrm{Pb}$ e $\mathrm{Cu}$, por exemplo), fácil e rápido crescimento celular e capacidade de crescer em meios com altas concentrações de $\mathrm{CO}_{2}$ (Lehninger, 1990; Lourenço, 2006; Huntley, 2007).

Apesar de ser um processo ainda pouco explorado em grande escala, a obtenção de energia a partir das microalgas ganha adeptos em muitas partes do mundo, como nos Estados Unidos, Alemanha, Israel, Bélgica, França e Nova Zelândia, os quais desenvolvem pesquisas nesta área. $\mathrm{O}$ incentivo à utilização de microalgas como fonte de matéria prima para produção de biocombustíveis (como o biodiesel, o biogás e o bioetanol), é decorrente dos grandes avanços da engenharia de biossistemas nesse meio, mas que ainda apresenta grandes lacunas a serem otimizadas. Além de outros desafios, a utilização de biorreatores contínuos para o crescimento celular ainda é tido como um campo relativamente pouco estudado. O cultivo em modo de operação contínuo é de grande vantagem, quando comparado aos modos em batelada e semi batelada, em números em produtividade celular (Richmond, 2004; Lourenço, 2006).

Numa linha de pesquisa desse tipo, o comportamento reológico ocupa posição de destaque, pois todos os materiais envolvidos no processo têm comportamento não newtoniano e devem ser submetidos a todo tipo de operações unitárias como agitação, mistura, trocas de calor, movimentação por bombas especiais, separações e outras que podem advir da concepção e desenvolvimento de todo o processo (Lira, 2011).

Das observações anteriores, pode-se notar a importância de criteriosa caracterização reológica para os diferentes tipos de alimentação em reatores, já que os dados de viscosidade usados nos projetos de equipamentos devem ser determinados com a máxima precisão possível, evitando-se um sub ou superdimensionamento. Ademais, os dados reológicos são de fundamental importância na economia de energia, o que nas últimas décadas se tornou extremamente importante a ponto de cada vez mais as operações de troca de calor e massa serem encaradas de forma mais minuciosa (Queiroz, 1998; Pelegrine, 1999; Silva et al., 2005).

Considerando-se a escassez de informações a respeito de dados reológicos na literatura científica, o presente trabalho teve como objetivo analisar o comportamento reológico de suspensões da microalga Chlorella $s p$ em diferentes tempos de cultivo, em reatores dos tipos batelada, assim como obter os parâmetros reológicos das mesmas, de modo a estimar as demandas energéticas de cada etapa, visando à otimização na construção de um biorreator tubular de alimentação contínua para o cultivo celular desta microalga. 


\section{MATERIAIS E MÉTODOS}

Para o estudo em questão, foi utilizada a microalga Chlorella $s p$, oriunda de Cabo Frio, RJ, (chlor-CF), que foi gentilmente doada pelo Departamento de Oceanografia Biológica do Instituto Oceanográfico da Universidade de São Paulo.

O trabalho teve como objetivo analisar o comportamento reológico de suspensões da microalga Chlorella $s p$ em diferentes tempos de cultivo, em reatores do tipo batelada, de modo a otimizar todas as etapas da produção do biodiesel, a partir da microalga Chorella $s p$, desde o seu cultivo em fotobiorreatores.

\subsection{Cultivo das microalgas para a produção do biodiesel:}

Para cada série de cultivo, todo o material de vidraria a ser usado foi lavado em água corrente, rinsado com água destilada e autoclavado a $121^{\circ} \mathrm{C}$ durante 20 minutos. Para os erlenmeyers de ensaio, foram utilizados como tampa pedaços de papel alumínio (Xia et al., 2011).

A inoculação das microalgas foi feita próximo à chama de lamparina, e o cultivo, em sala climatizada. A iluminação foi feita com lâmpadas fluorescentes de 40 W. A intensidade luminosa foi medida com um luxímetro (AKSO, modelo AK 308) e a temperatura da sala mantida constante por um aparelho de ar-condicionado tipo quente/frio. $O$ cultivo foi feito numa adaptação no Laboratório do Meio Ambiente, coordenado pelo prof. Messias B. Silva, na qual consta uma sala, de aproximadamente $8 \mathrm{~m}^{2}$, isolada de luz difusa e portada de um aparelho de arcondicionado portátil quente/frio, para controle total de luz artificial e de temperatura.

Todos os reagentes utilizados na preparação dos meios de cultivo foram de padrão analítico, e o meio de cultivo básico, para manutenção do cultivo mãe, foi da seguinte composição: sal marinho (1 g/L), $\mathrm{NaNO}_{3} \quad(75 \mathrm{~g} / \mathrm{L}), \quad \mathrm{NaH}_{2} \mathrm{PO}_{4} \cdot \mathrm{H}_{2} \mathrm{O} \quad(5 \mathrm{~g} / \mathrm{L})$, $\mathrm{FeCl}_{3} \cdot 6 \mathrm{H}_{2} \mathrm{O}(3,15 \mathrm{~g} / \mathrm{L}), \mathrm{Na}_{2}$ EDTA $(4,3 \mathrm{~g} / \mathrm{L})$, $\mathrm{ZnSO}_{4} \cdot 7 \mathrm{H}_{2} \mathrm{O}(22,2 \mathrm{mg} / \mathrm{L}), \mathrm{MnCl}_{2} \cdot 4 \mathrm{H}_{2} \mathrm{O}(180$ $\mathrm{mg} / \mathrm{L}), \mathrm{Na}_{2} \mathrm{MoO}_{4} \cdot 2 \mathrm{H}_{2} \mathrm{O} \quad(6,3 \quad \mathrm{mg} / \mathrm{L})$, $\mathrm{CoCl}_{2} \cdot 6 \mathrm{H}_{2} \mathrm{O} \quad(10 \mathrm{mg} / \mathrm{L}), \mathrm{CuSO}_{4} \cdot 5 \mathrm{H}_{2} \mathrm{O} \quad(9,8$ $\mathrm{mg} / \mathrm{L})$, Tiamina (100 mg/L), Cianocobalamina $(0,5 \mathrm{mg} / \mathrm{L})$ e Biotina $(0,5 \mathrm{mg} / \mathrm{L})$ (Guillard, 1975).

As soluções de estoque, utilizadas na preparação do meio de cultivo foram autoclavadas a $110{ }^{\circ} \mathrm{C}$, com exceção da solução de vitaminas (Tiamina, Cianocobalamina e Biotina), às quais foram filtradas através de filtros de $0,22 \mu \mathrm{m}$ de tamanho de poro.

A cepa da microalga Chlorella sp. utilizada nesse estudo foi mantida em uma incubadora confeccionada em madeira e dotada de um fotoperíodo, controlada por um temporizador. A intensidade luminosa fornecida foi de $15 \mathrm{~W}$ por uma lâmpada fluorescente.

Para manutenção de um banco de células, foi feita a repicagem em erlenmeyers de volume de $125 \mathrm{~mL}$, com volume útil de $100 \mathrm{~mL}$, com fotoperíodo de $12 \mathrm{~h}: 12 \mathrm{~h}$ (luz/escuro) e luminosidade média de 4,8 klux. As repicagens foram feitas em períodos de 10 a 15 dias, na proporção de $10 \mathrm{~mL}$ de cultura antecessora para $90 \mathrm{~mL}$ de meio de cultivo novo, e os frascos foram agitados de forma manual uma vez ao dia.

O crescimento microalgal foi acompanhado por análise de absorbância em espectrofotômetro UV-Vis modelo Bel Photonics, selecionando o comprimento de onda de $570 \mathrm{~nm}$ para a realização das leituras, de acordo com Lourenço (2006). Montou-se uma curva analítica relacionando a concentração de biomassa seca $(\mathrm{g} / \mathrm{L})$ com a absorbância a $570 \mathrm{~nm}$, onde o desenvolvimento da microalga foi analisado sob diferentes condições de temperatura, salinidade, volume da suplementação e 
concentração mássica de gás carbônico, nitrato e fosfato, conforme a tabela 1 .

Tabela 1: Faixa dos diferentes fatores ambientais no cultivo da microalga.

\begin{tabular}{|c|c|c|c|c|}
\hline Fator & Parâmentro & & Nivél Baixo (-) & Nível Alto (+) \\
\hline $\mathbf{A}$ & $\mathrm{CO}_{2}$ & $(\%)$ & $0 \quad(\%)$ & $2(\%)$ \\
\hline B & $\mathrm{NO}_{3}{ }^{-}$ & $(\mathrm{g} / \mathrm{L})$ & $0,25 \quad(\mathrm{~g} / \mathrm{L})$ & $0,50 \quad(\mathrm{~g} / \mathrm{L})$ \\
\hline C & $\mathrm{PO}_{4}^{-3}$ & $(\mathrm{~g} / \mathrm{L})$ & $0,35 \quad(\mathrm{~g} / \mathrm{L})$ & $0,65 \quad(\mathrm{~g} / \mathrm{L})$ \\
\hline D & Suplementação & $(\mathrm{mL})$ & $1 \quad(\mathrm{~mL})$ & $2(\mathrm{~mL})$ \\
\hline $\mathbf{F}$ & Temperatura & $\left({ }^{\circ} \mathrm{C}\right)$ & $20 \quad\left({ }^{\circ} \mathrm{C}\right)$ & $30 \quad\left({ }^{\circ} \mathrm{C}\right)$ \\
\hline G & Salinidade & $(\mathrm{g} / \mathrm{L})$ & $15 \quad(\mathrm{~g} / \mathrm{L})$ & $30 \quad(g / L)$ \\
\hline
\end{tabular}

A análise do desenvolvimento da microalga foi feita em oito diferentes condições ambientais, representadas na tabela 2 .

Tabela 2 - Condições dos diferentes fatores ambientais no cultivo da microalga.

\begin{tabular}{lccccccc}
\hline Teste & $\mathrm{CO}_{2}$ & $\mathrm{NO}_{3}$ & $\mathrm{PO}$ & Suplem. & Temperatura & Salinidade \\
\hline $\mathbf{1}$ & + & + & - & + & - & - \\
\hline 2 & + & + & - & - & + & + \\
\hline 3 & + & - & + & + & - & + \\
\hline 4 & + & - & + & - & + & - \\
\hline 5 & - & + & + & - & - & + \\
\hline 6 & - & - & - & + & + & +
\end{tabular}

\subsection{Análises reológicas do material:}

As medidas experimentais foram feitas no reômetro Brookfield modelo LVDV-3T, com temperatura controlada por um banho termoestático que circula na camisa onde se insere o recipiente destinado a armazenar o fluido durante o ensaio.

Os ensaios foram realizados em triplicata a $25^{\circ} \mathrm{C}$, sendo utilizada uma nova amostra para cada repetição. O sistema de medidas utilizado nesta determinação foi o de cilindros concêntricos, o qual consta de dois cilindros, sendo que apenas um deles gira à uma certa velocidade angular, enquanto o outro permanece imóvel. Este aparelho mantém uma velocidade de rotação constante que corresponde a certa taxa de deformação, e a tensão de cisalhamento é obtida através da medida do torque no cilindro de medida, que se mantém fixo. Consequentemente, estabelecendo-se várias velocidades angulares para o cilindro rotacional e detectando-se o torque correspondente no cilindro de medida, as curvas reológicas podem ser obtidas, para determinado fluido. Como nos ensaios iniciais constatou-se que a suspensão da microalga Chlorella sp apresentou comportamento reológico de um fluido não Newtonioano, julgou-se interessante a análise da variação da tensão de cisalhamento em função da taxa de deformação. Para isto, utilizou-se uma haste tipo cilíndrica coaxial SC4-31, compatível com os acessórios Thermosel e adaptador para pequenas amostras SSA com câmara SC413R, nos reômetros das séries LV, em aço inoxidável \# 304. Neste adaptador, utiliza amostras de 10,0 ml.

O instrumento dispõe de software, no qual torna-se possível programar os intervalos de tempo entre cada leitura, registrando dados de taxa de deformação, tensão de cisalhamento, viscosidade e temperatura.

Os resultados obtidos foram processados com o software ORIGIN 7.0, e as curvas obtidas ajustadas aos modelos reológicos de Casson, Ostwald-de-Waelle (Lei da Potência) e Bingham, sendo também determinado o grau de tixotropia do material, além dos seguintes parâmetros estatísticos:

$\mathrm{R}^{2}$ - coeficiente de determinação mede a proporção da variação total da média explicada pela regressão, definido como a soma quadrática total.

$$
X^{2} \text { - qui-quadrado - expressa a }
$$
diferença entre os valores previstos pelo modelo e os valores obtidos experimentalmente.

A verificação da existência de tixotropia foi feita através da avaliação das curvas de escoamento obtidas, variando-se a taxa de deformação (ou a tensão de cisalhamento), durante um período de tempo, 
mantendo-se a temperatura constante. Após o ensaio, se o fluido for tixotrópico ou reopéctico, nota-se a presença da histerese: quando a curva relativa ao aumento da taxa de deformação não coincide com a curva de decréscimo da mesma, no reograma.

A quantificação do valor da tixotropia foi feita através de leitura direta no equipamento, o qual foi programado para seguir as etapas propostas por Nascimento (2007) e seguidas por Shiroma (2012), as quais consistem em:
i. Aumentar a taxa de deformação de 0 a $\gamma_{M}$ por um período $\mathrm{t}_{1}$;
ii. Manter a taxa de deformação $\gamma_{\mathrm{M}}$ por um período $\mathrm{t} 2$;
iii. Reduzir a taxa de deformação, de $\gamma_{M}$ para 0 por um período $t 1$.

\section{RESULTADOS E DISCUSSÕES}

Os gráficos da viscosidade aparente em função da taxa de deformação obtidos nas diferentes condições do experimento encontram-se na Figuras 1. Nessa figura, os resultados apresentados são as médias das triplicatas experimentais

Figura 1 - Viscosidade aparente da suspensão de Chlorella sp, nas diferentes condições de processo.

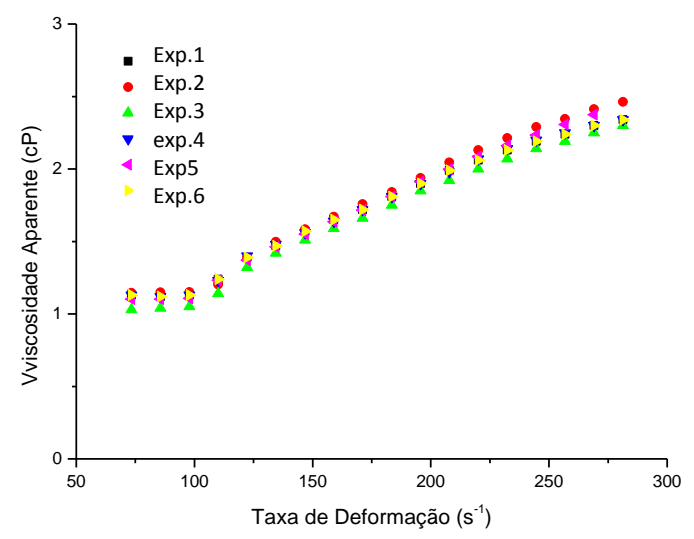

Da Figura 1 observa-se que, para a suspensão da microalga Chlorella sp, obtida a partir de diferentes condições de processo, a sua viscosidade aparente aumentou o aumento na frequência de rotação (a qual é diretamente proporcional à taxa de deformação), em todos os casos. Por outro lado, durante a realização das três repetições para medidas de viscosidade, para a mesma condição de processo, pôde-se observar que os valores das viscosidades aparentes não foram tão próximos, o que indica que há indícios de que tal suspensão apresenta uma certa tixotropia, ou seja, a sua viscosidade aparente diminuiu com o tempo, para uma dada frequência de rotação, possivelmente devido à sedimentação das partículas em suspensão. Tal observação foi comprovada ao analisar o grau de tixotropia da suspensão da microalga Chlorella sp: para todas as condições de processo, $\mathrm{p}$ índice de tixotropia resultou no valor de 0,5 . Estes resultados estão coerentes com diversos autores, para suspensões de algas em geral: as mesmas apresentam comportamento reológico de fluido dilatante e, uma explicação para este tipo de comportamento é que, à medida que a tensão de cisalhamento aumenta, o líquido intersticial que lubrifica a fricção entre as partículas é incapaz de preencher os espaços, devido a um aumento no volume que frequentemente acompanha $\mathrm{o}$ fenômeno, ocorrendo contato direto entre as partículas sólidas, fazendo com que a viscosidade aparente aumente (Tischer, 2006; Brangel, 2011). Poucos estudos que discutem as propriedades reológicas de hidrocolóides, sendo a grande maioria dos trabalhos realizados com a kappa-carragenana (Tecante e Doublier, 2002; Tester e Sommerville, 2003; Verbeken et al., 2004; Shi e Bemiller, 2002; Tye, 1988).

Com relação às oito diferentes condições de processo, a Figuras 1 mostra que 
há uma certa tendência do fluido tornar-se menos viscoso quando a suspensão da microalga Chlorella sp for tratada com baixos teores de $\mathrm{NO}_{3}$, suplementação e menor salinidade do meio (experimentos 3 e 8). Por outro lado, observa-se na Figura 1 que as curvas estão próximas umas das outras, o que sugere que as diferentes condições de processo pouco influenciam na viscosidade da suspensão de Chlorella sp, independente do tratamento.

A Tabela 3 apresenta os valores dos parâmetros obtidos através do ajuste dos modelos de Bingham, Casson e Lei da Potência aos reogramas correspondentes à Figura 2, para as condições de processo correspondentes aos experimentos 1, 2, 3, 4, 6 e 7. Nesta tabela, cada parâmetro corresponde ao valor da média dos valores nos diferentes tempos de cultivo e os valores entre parênteses, o desvio padrão.

Tabela 3: Parâmetros reológicos da suspensão

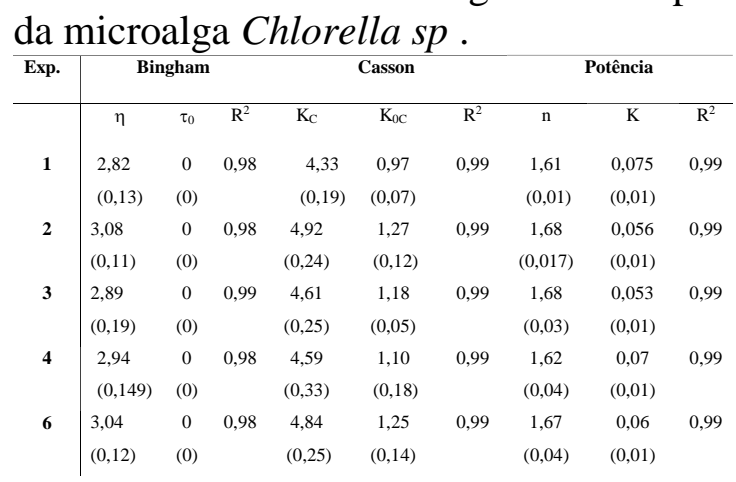

Da tabela 1 pode-se observar que tanto o modelo de Bingham quanto os de Casson e Ostwald-de-Waelle (Lei da Potência) proporcionaram bons parâmetros de ajuste, apresentando elevados para o coeficiente de determinação $\left(r^{2}\right)$. Os resultados apresentados nas tabelas reforçam a teoria de que a suspensão de microalgas Chlorella $s p$ apresenta comportamento reológico de fluidos dilatantes, visto que os valores dos índice de comportamento foram superiores a 1. Além disto, os valores da tensão inicial foram nulos quando os dados foram ajustados ao modelo reológico de Bingham; quando ajustados ao modelo reológico de Casson, tais valores foram próximos a zero. Neste ponto, vale ressaltar que as condições de processo não influenciaram no tipo de comportamento reológico, apesar de exercer efeito no índice de consistência.

\section{NOMENCLATURA}

$\eta \Rightarrow$ Viscosidade Plástica (cP)

$\tau_{0} \Rightarrow$ Tensão inicial (dyne $/ \mathrm{cm}^{2}$ )

$\mathrm{K}_{\mathrm{C}} \Rightarrow$ Viscosidade Plástica de Casson $\left(\right.$ dyne $\left./ \mathrm{cm}^{2}\right)$

$\mathrm{K}_{0 \mathrm{C}} \Rightarrow$ Tensão inicial de Casson

$\mathrm{n} \Rightarrow$ índice de comportamento

$\mathrm{K} \Rightarrow$ índice de consistência (cP)

\section{CONCLUSÃO}

Dos resultados apresentados no item 3, pode-se concluir que a suspensão da microalga Chlorella $\mathrm{sp}$, para qualquer condição de processo, apresentou comportamento reológico típico de um fluido dilatante, embora a suspensão tenha apresentado uma certa tixotropia.

Ademais, o tempo de processamento praticamente não exerceu influência nos reogramas.

\section{REFERÊNCIAS}

ALBUQUERQUE, G.A.; CONCEIÇÃO, M.M.; SILVA, M.C.D.; SANTOS, I.M.G.; FERNANDES,V.J.; SOUZA, A.G. Avaliação reológica e caracterização físico-química do biodiesel de canola e misturas. In: Anais do Congresso da Rede Brasileira de Tecnologia de Biodiesel, Brasília. RTBT, 175-179, 2006. 
AZEREDO, V.B.S. Produção de biodiesel a partir do cultivo de microalgas: estimativa de custo e perspectivas para o Brasil. 2012. 171p. Dissertação (Mestrado em Planejamento Energético, Universidade Federal do Rio de Janeiro.

BARBOSA, A.L.P.B. Biodiesel de mamona em trator agrícola na operação de preparo do solo. 2007. 75p. Tese (Doutorado em Engenharia Agrícola). Universidade Estadual Paulista.

BASHA, S.A.; GOPAL, K.R.; JEBARAJ, S. A review on biodiesel production, combustion, emissions and performance. Renewable and Sustainable Reviews, v. 13, p. 1628-1634, 2009.

COSTA NETO, P.R.; ROSSI, L.F.S.; ZAGONEL, G.F.; RAMOS, L.P. Produção de biocombustível alternativo ao óleo diesel através da transesterificação de óleo de soja usado em frituras. Química Nova, v.23, n.4, p.531-537, 2000.

DANTAS, M.B. Obtenção, caracterização e estudo termoanalítico de biodiesel de milho. 2006. 205p. Dissertação (Mestrado em Química), Universidade Federal da Paraíba, João Pessoa.

DOGARIS, I.; GKOUNTA, O.; MAMMA, D.; KEKOS, D. Bioconversion of dilute-acid pretreated bagasse to etanol by Neusorpora crassa. Applied Microbiology and Biotechnology, v.95, n.2, p.541-550, 2013.

FITZPATRICK, M.; CHAMPAGNE, P.; CUNNIGHAM, M.F.; WHITNEY, R.A. A biorrefinary processing perspective: treatment lignocellulosic materials for the production of value-add products. Bioresource Technology, v.101, n.23, p.8915-8922, 2010.
FERRARI, A.R.; OLIVEIRA, V.S. SEABIO, A. Biosiesel de soja - taxa de conversão em ésteres etílicos, caracterização físico-química e consumo em gerador de energia. Química Nova, v.28, n.1, p.19-23, 2005.

GUILLARD, R. R. L. Culture of phytoplankton for feeding marine invertebrates. In: SMITH, W. L.,

CHANLEY, M. H. (Eds.). Culture of marine invertebrate animals. New York: Plenum, 1975.

HUNTLEY, M. E., REDALJE, D. G. $\mathrm{CO}_{2}$ mitigation and renewable oil from photosynthetic microbes: a new appraisal. Mitigation and Adaptation Strategies for Global Change, v.12, p.573-608, 2007.

LEHNINGER, A. L. Princípios de Bioquímica. São Paulo: Savier, 1990.

LIRA, R.A. Estudo do rendimento de biomassa da microalga nativa Chlorella $s p$ visando a obtenção de biocombustíveis. 2011. 119p. Tese (Doutorado em Engenharia Agrícola), Universidade Federal de Viçosa.

LOURENÇO, S. O. Cultivo de microalgas marinhas: princípios e aplicações. São Carlos: RiMa. 2006.

NASCIMENTO, C.R. Ensaios em reômetro rotacional tipo cilindros coaxiais. Comunicação Técnica elaborada para o livro tratamento de Minérios: Práticas laboratoriais - Centro de tecnologia Mineral.

PELEGRINE, D.H. Comportamento reológico das polpas de manga e abacaxi. 1999. 79p. Dissertação (Mestrado em Engenharia de Alimentos) - Universidade Estadual de Campinas. 
QUEIROZ, A.J. Análise do comportamento reológico dos sucos de manga e abacaxi. 1998. 109p. Tese (Doutorado em Engenharia de Alimentos). Universidade Estadual de Campinas.

RICHMOND, A. Handbook of microalgae culture: biotechnology and applied phycology. Oxford: Blackwell Science, 2004.

SHARMA, Y.C. SINGH, B.; UPADHYAY, S.N. Advancements in development and characterization of biodiesel: a review. Fuel, v.87, n.12, p.2355-2373, 2008.

SHI, X.; BeMILLER, J.N. Effects of food gums on viscosities of starch suspensions during pasting. Carbohydr. Polymers., v. 50, p. 7-18, 2002.

SHIROMA, P.H. 2012. 130p. Estudo do comportamento reológico de suspensões aquosas de bentonita e CMC: Influência da concentração do $\mathrm{NaCl}$. Dissertação (Mestrado em Engenharia Química) - Universidade de São Paulo.

SILVA， F， C; PELEGRINE， D.H.G.; GASPARETTO, C.A. Reologia do suco de acerola:efeitos da concentração e da temperatura. Revista da Sociedade Brasileira de Ciência e Tecnologia de Alimentos, v.25, n.1, p. 121-126, 2005.

SILVA FILHO, J.B. Produção de biodiesel etílico de óleos e gorduras residuais (ogr) em reator químico de baixo custo. 2010. 57p. Dissertação (Mestrado em Engenharia Mecânica e de Materiais), Universidade Tecnológica Federal do Paraná.

TECANTE A.; DOUBLIER, J.L. Rheological investigation of the interaction between amylase and $\kappa$-carrageenan. Carbohydr. Polymers., v. 49, p. 177-183, 2002.
TESTER, R.F.; SOMMERVILLE, M.D. The effects of non-starch polysaccharides on the extent of gelatinization, swelling and $\alpha$ amylase hydrolysis of maize and wheat starches. Food Hydrocol., v. 17, p. 41-54, 2003.

TISCHER, P.C.S. Estrutura química, propriedades reológicas e atividade antiviral das galactanas sulfatadas das algas vermelhas Meristiella gelidium e Gymnogongrus (gigartinales). 2006. 174p. Dissertação (Mestrado em Bioquímica e Biologia Molecular, Universidade Federal do Paraná.

TYE, R.J. The rheology of starch/carrageenan systems. Food Hydrocol., v. 4, p. 259-266, 1988.

VERBEKEN, D.; THAS, O.; DEWETTINCK, K. Textural properties of gelled dairy desserts containing $\kappa$-carrageenan and starch. Food Hydrocol., v. 18, p. 817823, 2004.

XIA, C., ZHANG, J., HU, B. A new cultivation method for microbial oil production: cell pelletization and lipid accumulation by Mucor circinelloides. Biotechnology for biofuels, v.4, p.4-15, 2011.

\section{AGRADECIMENTOS}

À Fapesp. 